\title{
Pirólisis de residuos de bioplásticos: Productos obtenidos del ácido poliláctico (PLA)
}

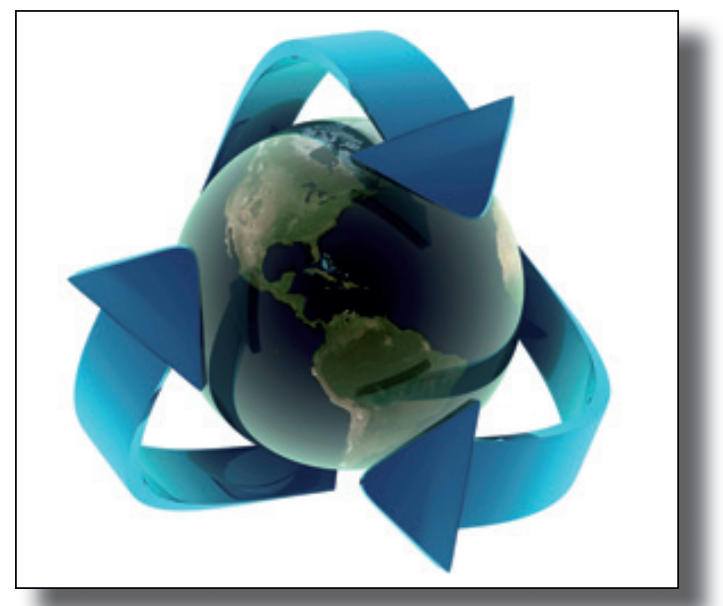

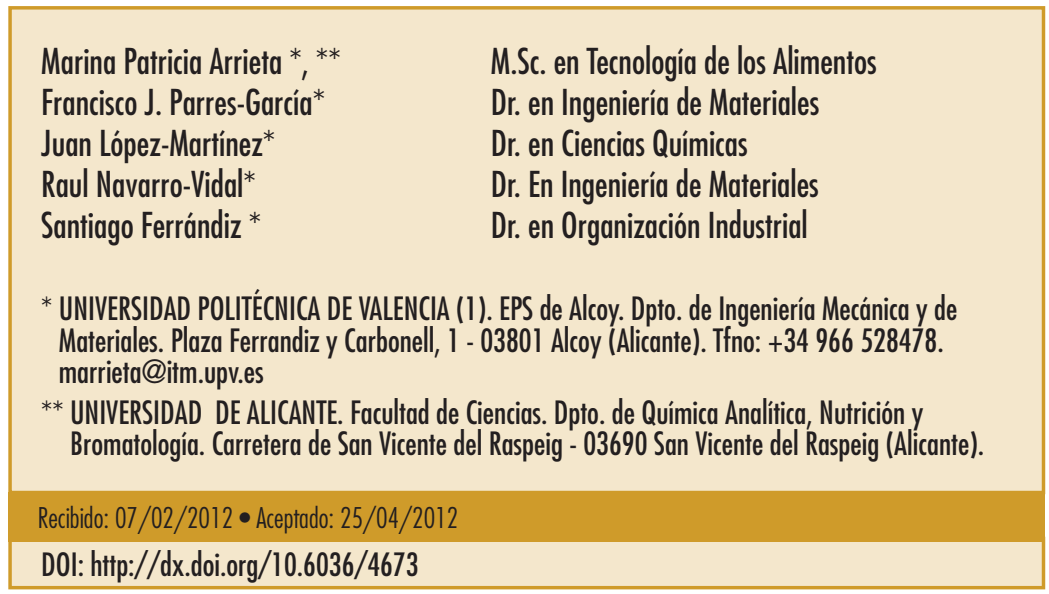

\section{PYROLYSIS OF BIOPLASTICS WASTE: Obtained products from Poly(Lactic acid) (PLA)}

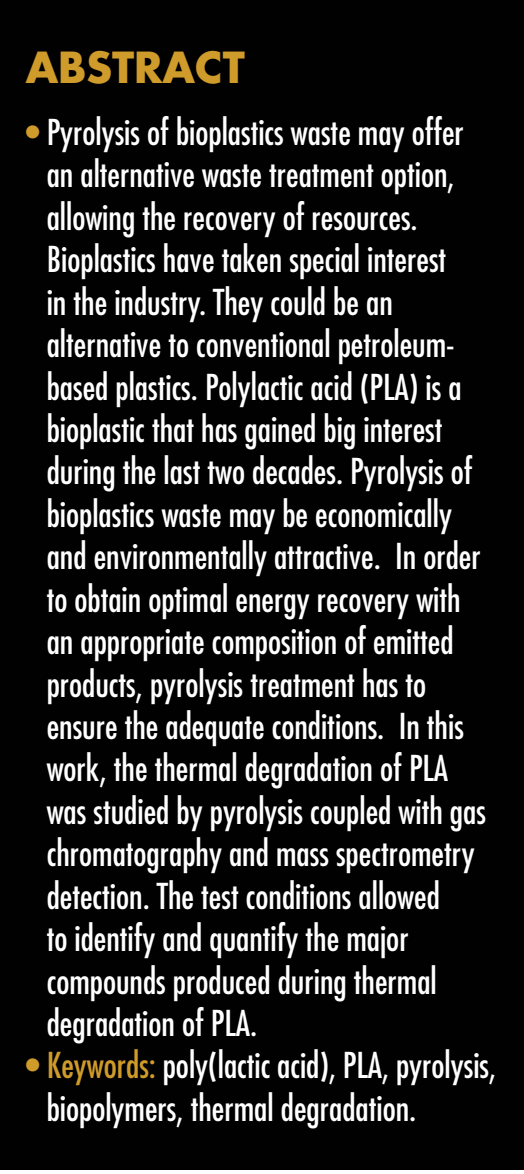

\section{RESUMEN}

El tratamiento de residuos de bioplásticos por pirólisis representa una alternativa que permite la recuperación de recursos. Los bioplásticos han cobrado especial interés en la industria debido a que representan una alternativa a los polímeros convencionales derivados del petróleo. El ácido poliláctico (PLA) es un biopolímero que ha cobrado gran interés en las últimas décadas. La pirólisis de residuos de bioplásticos puede resultar económica y medioambientalmente atractiva. Para ello, debe asegurar una óptima recuperación energética y una adecuada composición de los productos emitidos. En el presente trabajo se ha estudiado la degradación térmica del PLA mediante la técnica de pirolisis acoplada a cromatografía de gases con detección por espectrofotometría de masas (Py-GC/MS). Las condiciones ensayadas permitieron identificar los principales productos generados durante la degradación térmica de muestras de PLA.
Palabras clave: ácido poliláctico, PLA, pirolisis, biopolímeros, degradación térmica.

\section{1.- INTRODUCCIÓN}

Los bioplásticos han cobrado gran interés en los últimos años debido a que representan una alternativa prometedora para minimizar el impacto ambiental que generan los plásticos sintéticos, transcurrida su vida útil. Uno de los bioplásticos que mayor crecimiento ha generado en los últimos años es el ácido poliláctico (PLA). El cual se encuentra disponible en el mercado en aplicaciones plásticas prácticas como cubiertos, platos, tazas, tapas, pajitas de bebidas, bolsas, films, y algunas aplicaciones de envasado de alimentos $[1,2]$.

El PLA es un bipoliéster obtenido de la polimerización del ácido láctico, producidos por fermentación $\mathrm{o}$ síntesis química [3]. Es un polímero termoplástico biodegradable, que 
puede obtenerse a partir de fuentes renovables [4,5], o productos agrícolas simples como el maíz $[3,6]$. En las dos últimas décadas, el PLA ha cobrado especial interés debido su disponibilidad en el mercado, bajo coste, y su potencial para remplazar a otros polímeros sintéticos de uso común derivados del petróleo [4].

El impacto que produce un producto en el medio ambiente debe evaluarse desde la obtención de las materias primas o generación de recursos naturales hasta la eliminación [7]. La Directiva 94/62/CE del Parlamento Europeo y del Consejo, de 20 de diciembre de 1994, relativa a los envases y residuos de envases en su Anexo $I I$ inciso $d$, establece que los residuos de envases biodegradables deberán tener unas características que les permitan sufrir descomposición física, química, térmica o biológica de modo que la mayor parte del compost final se descomponga en último término en dióxido de carbono, biomasa y agua [8]. Así, los productos de materiales biodegradables, en lugar de ser desechados con el resto de materiales plásticos, pueden ser tratados junto con los residuos orgánicos. Uno de los destino de estos productos de PLA, tras su uso, es incorporarse a la corriente de biomasa. De esta manera, el PLA puede ser recuperado a través de procesos mecánicos (trituradotransformación térmica) o químicos (hidrólisis), o bien ser depositado junto con la materia orgánica para la formación de compost [9]. Los productos plásticos para demostrar que son compostables deben estar certificados. En la Tabla 1 se muestran las normativas por certificar a los materiales plásticos como compostables y los símbolos utilizados para identificar dichos productos. valorización de la biomasa y los residuos orgánicos [11].

El uso de la biomasa de los materiales se puede ampliar a nuevas aplicaciones. Por ejemplo, la biomasa puede ser utilizada como materia prima renovable en la producción de materiales orgánicos sintéticos, tales como productos químicos básicos, plásticos, pinturas y disolvente. La utilización de la biomasa como fuente de energía tiene que estar basada en estrategias de sostenibilidad, donde se consuma como máximo aquello que se produce [12]. Así, la pirólisis de la biomasa resulta una de las rutas de conversión de residuos más prometedoras ya que ofrece algunas ventajas como la reducción de costos, integración en el sistema de energía y beneficios medioambientales [11]. De esta manera, la aplicación de la tecnología de pirólisis al tratamiento de residuos ha ganado aceptación en la industria. La pirólisis de la biomasa puede ser descrita como la descomposición térmica directa del material, la cual permite reducir las emisiones de $\mathrm{CO}_{2}$ provenientes del tratamiento térmico de residuos debido a que se realiza en ausencia de oxígeno.

Existen aún pocas instalaciones en España en operación de pirólisis de residuos y estas se encuentran enfocadas a la valorización de neumáticos, a través de reducción de residuos de neumáticos por el proceso de pirólisis.

Sin embargo, la pirólisis de residuos de PLA puede ser económica y medioambientalmente atractiva [2]. Para ello, se requiere un mayor conocimiento de la descomposición térmica del PLA, ya que este material está siendo introducido a la industria a gran escala. Desde el punto de vista medioambiental, resulta preciso actuar no sólo en la fabricación de materiales provenientes de recursos

\begin{tabular}{|l|l|l|l|}
\hline Procedencia & \multicolumn{1}{|c|}{ Organización } & \multicolumn{1}{|c|}{ Normativas } & Símbolo de compostaje \\
\hline Europa & European Bioplastic & $\begin{array}{l}\text { EN 13432: 2001/AC 2005 } \\
\text { EN 14995:2007 }\end{array}$ & \\
\hline Estados Unidos & $\begin{array}{l}\text { Biodegradable Products } \\
\text { Institute }\end{array}$ & ASTM D6400-04 \\
ASTM D6868-11 & COMPOSTABLE \\
\hline
\end{tabular}

Tabla 1: Certificación de Plásticos compostables

Además de los estudios intensivos existentes de la biodegradabilidad del PLA en condiciones de compostaje, la estabilidad térmica y el comportamiento durante la degradación térmica cobran importancia para la transformación, aplicación, y el reciclado térmico [10] de este material. El tratamiento térmico de residuos puede ser por incineración, es decir en presencia de oxígeno; o bien por pirolisis, en ausencia de oxígeno (o con una cantidad limitada de éste) a altas temperaturas para generar una combustión directa.

La pirólisis representa una opción interesante para la renovables, sino también en el momento del post consumo por lo que es necesario saber gestionar sus residuos.

La degradación térmica del PLA es muy compleja debido a que varias reacciones ocurren simultáneamente $[10,13]$ ya que depende de varios factores. Para identificar los compuestos producidos, la pirólisis es una técnica analítica mucho más sensible que las técnicas tradicionales de análisis térmico como termogravimetría (TGA), o calorimetría diferencial de barrido (DSC) [14,15]. La combinación de la pirólisis acoplada a cromatografía de gases y con detección por espectrometría de masas (Py-GC-MS), resulta 
una herramienta muy ventajosa para la caracterización de polímeros y sus aditivos [16]. De esta manera, la PyGC-MS resulta una técnica analítica muy útil para obtener información sobre las reacciones química de degradación de los polímeros inducidas por la temperatura, puede proporcionar información de la cinética de descomposición de los polímeros así como también de la composición de los productos producidos durante la degradación térmica [17].

En este trabajo, se han determinado las condiciones ideales de pirólisis (temperatura y tiempo) a escala de laboratorio con las cuales se obtuvieron cromatogramas que permitieron identificar los principales productos de la degradación térmica del PLA.

\section{MATERIALES Y METODOS}

\subsection{MATERIALES}

El Ácido Poliláctico (PLA) fue suministrado por NatureWorks. El cloroformo $(99,8 \%$ pureza) fue proporcionado por Sigma Aldrich, (Móstoles, España).

\subsection{PREPARACIÓN DE LAS MUESTRAS}

Para la preparación de la muestra de PLA patrón se disolvió $0,1 \mathrm{~g}$ de PLA pellet en $30 \mathrm{~mL}$ de cloroformo a temperatura ambiente bajo agitación magnética continua. Las muestras de PLA se prepararon disolviendo $0,1 \mathrm{~g}$ de film en $30 \mathrm{~mL}$ de cloroformo. $10 \mu \mathrm{L}$ de la solución obtenida se colocaron en un tubo de pirólisis de cuarzo y se dejó evaporar el solvente a temperatura ambiente durante 48 horas.

\subsection{METODOLOGÍA EXPERIMENTAL \\ Pirólisis- Cromatografía de gases- Espectrómetría de Masas (Py-GC-MS) \\ Para la pirólisis de las muestras se utilizó un pirolizador} (Py) "Pyroprobe 1000, CDS Analytical", (Oxford, Pensilvania) equipado con una interface (CDS 1500) con inyector manual. El pirolizador se acopló a un cromatógrafo de gases (GC) “6890N, Agilent Technologies”, (España S.L., Madrid, España). La columna cromatográfica empleada fue una HP-5 ms (30 m de longitud; 0,25 mm de espesor y con una fase estacionaria de $0,25 \mu \mathrm{m})$. El horno del GC fue programado a $40^{\circ} \mathrm{C}$ durante 2 minutos seguido de un aumento hasta $200{ }^{\circ} \mathrm{C}$ a $5{ }^{\circ} \mathrm{C} \cdot \mathrm{min}^{-1}$, temperatura a la cual se mantuvo durante 15 minutos, y luego la temperatura se incrementó hasta $300{ }^{\circ} \mathrm{C} \mathrm{a} 20^{\circ} \mathrm{C} \cdot \mathrm{min}^{-1}{ }^{\circ} \mathrm{C}$, donde se mantuvo durante $5 \mathrm{~min}$. Se utilizó helio como gas transportador con una relación de split 50:1. Para la detección se utilizó un detector de masas "5973, Agilent Technologies", (España S.L., Madrid, España). El detector selectivo de masa se programó para detectar masas entre 40 y $650 \mathrm{amu}$. Las muestras de PLA fueron pirolizadas a $600{ }^{\circ} \mathrm{C}$ durante $0,5 \mathrm{~s}$. Los productos de pirólisis fueron identificados obteniendo sus espectros de masas y por comparación de éstos con espectros encontrados en la bibliografía.

\subsection{ANÁLISIS ESTADÍSTICO}

Para el procesamiento de los datos se utilizó el software Instrument Data Analysis de Agilent Technology.

\section{RESULTADOS}

Con el objetivo de conseguir las condiciones más favorables de trabajo y teniendo en cuenta que los parámetros de pirólisis, como la temperatura y el tiempo, resultan un factor clave para la obtención de una buena separación analítica de los productos de degradación, inicialmente se seleccionaron como punto de partida condiciones de trabajo utilizadas por otros autores, las cuales han proporcionado buenos resultados [14]. Así, para las condiciones iniciales se mantuvo el tiempo de pirólisis constante a 2 s y se realizaron pirolisis a tres temperaturas diferentes. En las Figs. 1, 2 y 3 se muestran los cromatogramas obtenidos al pirolizar una muestra de PLA a temperaturas de $400^{\circ} \mathrm{C}, 500^{\circ} \mathrm{C}$ y $600^{\circ} \mathrm{C}$, respectivamente. Tras esta primera experiencia se observó, como era de esperar, un incremento de los compuestos en función de la temperatura de pirólisis utilizada, siendo la temperatura de $600{ }^{\circ} \mathrm{C}$ la que presentaba un cromatograma más detallado. Sin embargo, en la Fig. 3 se puede observar que a $600{ }^{\circ} \mathrm{C}$ se produjo saturación y solapamiento de

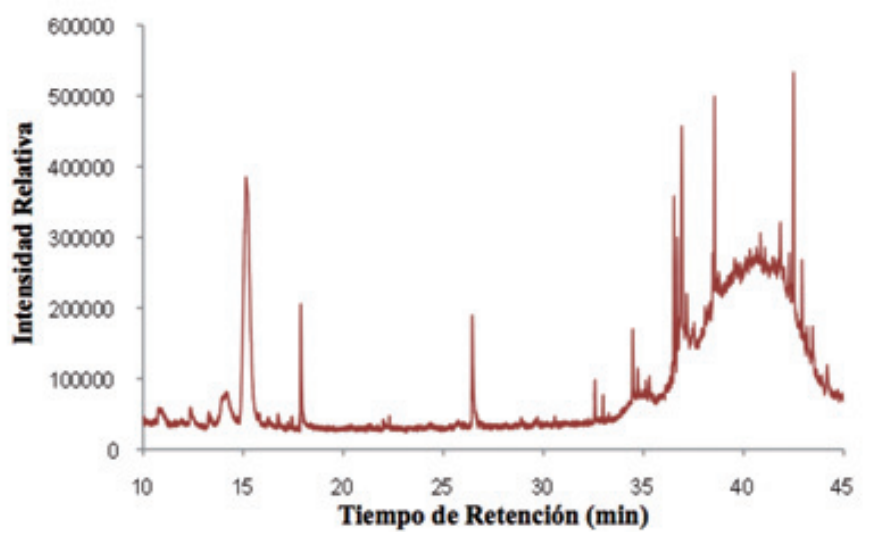

Fig. 1: Cromatograma obtenido de una muestra de PLA pirolizada a $400^{\circ} \mathrm{C}$ durante 2 s.

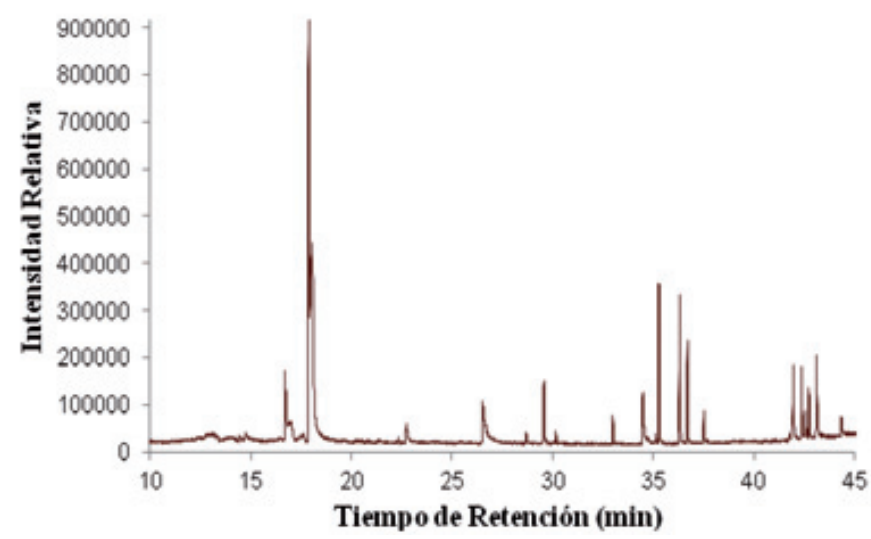

Fig. 2: Cromatograma obtenido de una muestra de PLA pirolizada a $500^{\circ} \mathrm{C}$ durante 2 s. 
algunos picos y por lo tanto dificultaba su identificación y cuantificación.

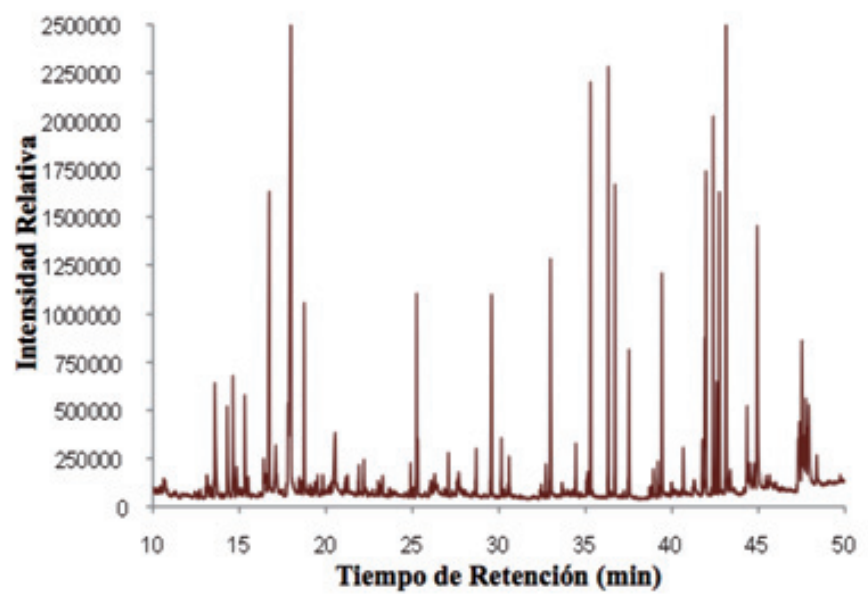

Fig. 3: Cromatograma obtenido de una muestra de PLA pirolizada a $600^{\circ} \mathrm{C}$ durante 2 s.

Con la finalidad de obtener una mejor separación de los picos, se modificó el tiempo de pirolisis. La utilización de tiempos cortos de pirolisis puede mejorar la separación cromatográfica de los productos de pirólisis [18], es por ello que en un segundo estudio se procedió a mantener constante la temperatura de pirólisis $\left(600{ }^{\circ} \mathrm{C}\right)$ y se redujo paulatinamente en tiempo de pirólisis $(1,5 \mathrm{~s} ; 1 \mathrm{~s}$ y $0,5 \mathrm{~s})$. Tras esta segunda experiencia, el cromatograma que presenta una adecuada separación de compuestos se obtuvo utilizando tiempo de 0,5 s (Fig. 4).

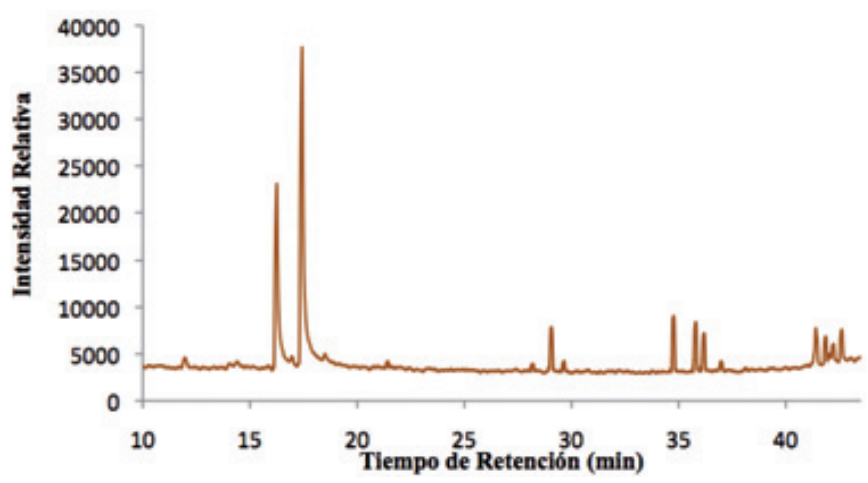

Fig. 4: Cromatograma obtenido de una muestra de PLA pirolizada a $600^{\circ} \mathrm{C}$ durante $0,5 \mathrm{~s}$.

Con el espectrómetro de masas se efectuó la identificación de los principales productos de pirólisis obtenidos. Las muestras mostraron la presencia de dos picos con espectro de masas muy similares, cuyos fragmentos principales fueron $\mathrm{m} / \mathrm{z}=32,43,45$, y 56 . De acuerdo con la literatura el primer pico corresponde a mesoláctida y el segundo a láctida $[19,20]$. El grupo de picos que aparecen a tiempos de retención superiores a 20 minutos fueron asignados a oligómeros cíclicos mayores que láctida [13,17-18], un dímero, tres trímeros y un tetrámero. La Tabla 2 muestra la lista de los principales productos formados durante la pirólisis de PLA a $600^{\circ} \mathrm{C}$ durante $0,5 \mathrm{~s}$.

\begin{tabular}{|l|c|}
\hline Producto de Pirólisis de PLA & Tiempo de Retención (min) \\
\hline Mesoláctida & 16,2 \\
\hline Láctida & 17,4 \\
\hline Dímero & 29,5 \\
\hline Trímero a & 34,6 \\
\hline Trímero b & 35,6 \\
\hline Trímero c & 36,7 \\
\hline Tetrámero & 42,4 \\
\hline
\end{tabular}

Tabla 2: Productos derivados de la pirólisis de PLA a $600^{\circ} \mathrm{C}$ durante $0,5 \mathrm{~s}$

Una vez optimizado el método de trabajo y seleccionadas las condiciones en las que se llevaría a cabo el análisis de productos de pirólisis de PLA se aplicó a 4 muestras (M1M4) preparadas a partir de films de PLA disueltos en cloroformo de cantidades conocidas comprendidas entre 0,35 y $1,00 \mu \mathrm{g}(\mathrm{M} 1=0,35 \mu \mathrm{g} ; \mathrm{M} 2=0,70 \mu \mathrm{g}, \mathrm{M} 3=1,00 \mu \mathrm{g}$ y M4=1,20). A partir de los resultados obtenidos de la pirólisis de la muestras de film de PLA se calculó la relación de áreas de los picos en función del área del pico de láctida (Tabla 3). La relación mesoláctida: láctida, ha sido utilizada por otros autores como una medida semi-cuantitativa de productos de pirolísis de PLA [20,21]. En el presente trabajo la relación de picos en función de se mantiene constante para los distintos tamaños de muestra, lo que nos permite inferir en la buena reproducibilidad del método.

\begin{tabular}{|l|c|c|c|c|}
\hline Relación & M1 & M2 & M3 & M4 \\
\hline Mesoláctida : Láctida & 7,84 & 7,81 & 7,42 & 7,63 \\
\hline Dímero : Láctida & 16,71 & 17,42 & 16,64 & 17,15 \\
\hline Trímero a: Láctida & 19,92 & 19,91 & 19,61 & 19,80 \\
\hline Trímero b : Láctida & 24,97 & 26,37 & 24,01 & 25,06 \\
\hline Trímero c : Láctida & 37,01 & 34,81 & 34,98 & 35,34 \\
\hline Tetrámero : Láctida & 62,65 & 62,70 & 58,91 & 61,75 \\
\hline
\end{tabular}

Tabla 3: Relación producto: láctida obtenidas en las muestras analizadas calculadas a partir de distintos productos de pirólisis obtenidos.

\section{DISCUSIÓN}

Para poder realizar el tratamiento de residuos de plásticos biodegradables por pirolisis, conjuntamente con el resto de residuos orgánicos, se requiere obtener una adecuada composición de los productos emitidos durante el proceso. En el presente trabajo se estudió el tratamiento de residuos de PLA mediante pirólisis a nivel de laboratorio. Se optimizó una metodología experimental para la identificación de los productos de pirólisis del PLA utilizando un pirolizador acoplado a un cromatógrafo de gases y un espectrómetro de masas. Con la optimización de los parámetros de pirólisis 
temperatura y tiempo, se logró una adecuada separación de todos los productos de degradación estudiados. Estas condiciones permitieron una correcta identificación de siete productos de degradación de PLA. Se observó que el PLA presentó una relación de mesoláctida: láctida de 1: 7,7 $\pm 0,2$. Khabbaz et al. (2000) [20] analizaron por pirólisis a $400^{\circ} \mathrm{C}$ durante 2s L-PLA degradado biológicamente en un medio biótico y obtuvieron una relación de mesoláctida: láctida de 1: 15 . A $500^{\circ} \mathrm{C}$ durante $2 \mathrm{~s}$ encontraron una relación de mesoláctida: láctida de 1: 2,4 en el mismo tipo de muestras. Con estas últimas condiciones de trabajo $\left(500^{\circ} \mathrm{C}-2 \mathrm{~s}\right)$ obtuvieron una relación de mesoláctida: láctida de 1: 4,7 en muestras hidrolizadas químicamente. Así, la relación de mesoláctida: láctida ha sido utilizada por estos autores como una medida semi-cuantitativa. La pirólisis representa una opción atractiva para la valorización de la biomasa y los residuos orgánicos. La viabilidad de la metodología de análisis desarrollada fue comprobada analizando muestras de films de PLA. De los resultados obtenidos (Tabla 3) se pudo observar que la relación de los distintos productos en función de láctida se mantiene constante independientemente del tamaño de la muestra. Se obtuvieron resultados satisfactorios, todas las muestras ensayadas mostraron que láctida es el principal producto obtenido durante la pirolisis de PLA. Estos resultados coinciden con los resultados obtenidos por otros autores [2]. Chien et al., (2011) [2] reportaron que la cantidad de hidrocarburos aromáticos poli-cíclicos emitidos a partir de la pirólisis de PLA es significativamente más baja que aquellas asociadas a otras formas de combustiones, lo que sugiere que la pirolisis resulta, dentro de los distintos procesos de combustiones, una manera apropiada para la eliminación de residuos de PLA.

\section{AGRADECIMIENTOS}

Los autores agradecen al Ministerio de Educación y Ciencia por el apoyo prestado a través del proyecto MAT2011-28468-C02-02 y al Profesor Alfonso Jiménez del Departamento deQuímicaAnalítica Nutrición y Bromatología de la Universidad de Alicante por su colaboración. Marina Patricia Arrieta ha sido becada por la Generalitat Valenciana para una beca para la formación de personal investigador en centros de investigación de la Comunitat Valenciana, dentro del programa Santiago Grisolía (GRISOLIA/2011/007).

\section{6.- BIBLIOGRAFÍA}

[1] Conn, R.E., Kolstad, J.J., Borzelleca, J.F., Dixler, D.S., Filer Jr L.J., Ladu Jr B.N., Pariza, M.W. "Safety assessment of polylactide (PLA) for use as a food contanct polymer". Food and Chemical Toxicology. 1995. Vol.33:4, p.273-283.

[2] Chien, Y.C., Liang, C., Yang, S.H. "Exploratory study on the pyrolysis and PAH emissions of polylactic acid". Atmospheric Environment. 2011. Vol.45, p.123-127. http://dx.doi.org/10.1016/j.atmosenv.2010.09.035
[3] Jamshidian, M., Tehrany, E.A., Imran, M., Jacquot, M., Desobry, S. "Production, Applications, Nanocomposites, and Release Studies". Comprehensive Reviews in Food Science and Food Safety. 2010.Vol. 9, p.552-571.

[4] Martino, V.P., Jiménez, A., Ruseckaite, R.A., Avérous, L. "Structure and properties of clay nano-biocomposites based on poly(lactic acid) plasticized with polyadipates" Polymers Advanced Technologies. 2010. DOI: 10.1002/pat.1747.

[5] Kulinski, Z, Piorkowska, E. "Crystallization, structure and properties of plasticized poly(L-lactide)". Polymer. 2005. Vol. 46, p.10290-10300. http://dx.doi.org/10.1016/j.polymer.2005.07.101

[6] Martino, VP, Ruseckaite, RA, Jiménez, A. "Ageing of poly(lactic acid) films plasticized with commercial polyadipates" Polymer International. 2009. Vol.58, p.437-444.

[7] Prado-Trigo A, Campo-Rámila F.,Munozgurien-Colindres J. "Aulas de Ecodiseño: análisis de ciclo de vida y ecodiseño en la industria". DYNA Ingeniería e Industria. Febrero 2011. Vol.86-1 p.59-73.

[8] Directiva 94/62/CE del Parlamento Europeo y del Consejo, de 20 de diciembre de 1994, relativa a los envases y residuos de envases. DOC $n^{\circ} \mathrm{L} 365 / 10,31.12 .94$

[9] Xuan P, Xiuli Z, Zhaohui T, Xuesi C. "Polylactic acid (PLA): Research development and industrialization" Biotechology Journal. 2010. Vol.5:11, p.1125-1136.

[10] Aoyagi, Y, Yamashita, K., Doi, Y. "Thermal degradation of poly[(R)3-hydroxybutyrate], poly[ع-caprolactone], and poly[(S)-lactide]". Polymer Degradation and Stability. 2002. Vol.76, p.53-59. http://dx.doi.org/10.1016/S0141-3910(01)00265-8

[11] Gómez-Días, C.L. Understanding Biomass Pyrolysis Kinetics: Improved Modeling Based on Comprehensive Thermokinetic Analysis. Chemical Engineer. Tesis Doctoral. Universitat Politècnica de Catalunya, Barcelona, 2006. ISBN: 978-84-6905558-8

[12] García-Sanz-Calcedo J, López-Rodriguez F, Cuadros-Blazquez F. "Eficiencia de una caldera de biomasa en un centro hospitalario" DYNA Ingeniería e Industria. Junio 2011. Vol.86-3 p.343-349.

[13] Kopinke D, Remmler M, Mackenzie K, Moder M, Wachsen 0 . "Thermal decomposition of biodegradable polyesters-II. Poly(lactic acid)". Polymer Degradation and Stability. 1996. Vol.53:3, p.329-342. http://dx.doi.org/10.1016/0141-3910(95)00221-9

[14] Parres, F., Balart, R., Crespo, J.E., López, J. "Effects of the injection-molding temperatures and pyrolysis cycles on the butadiene phase of high-impact polystyrene". Journal of Applied Polymer Science. 2007. Vol.106, p.1903-1908.

[15] Parres, F., Sánchez, L., Balart, R., López, J. "Determination of the photo-degradation level of high impact polystyrene (HIPS) using pyrolysis-gas chromatography-mass spectrometry". Journal of Analytical Applied Pyrolysis. 2007. Vol.78, p. 250-256. http://dx.doi.org/10.1016/j.jaap.2006.08.001

[16] Rial-Otero, R., Galesio, M., Capelo, J.L., Simal-Gádara, J. "A Review of Synthetic Polymer Characterization by Pyrolysis-GCMS". Chromatographia. 2009. Vol.70: 3/4, p.339-348.

[17] Kopinke FD, Mackenzie, K. "Mechanistic aspects of the thermal degradation of poly(lactic acid) and poly( $\beta$-hydroxybutyric acid)". Journal of Analytical and Applied Pyrolysis. 1997. Vol.40-41, p.43-53.

http://dx.doi.org/10.1016/S0165-2370(97)00022-3

[18] Moldoveanu SC. Instrumentation used for pyrolysis en Analytical Pyrolysis of Natural Organic Polymers. Elsevier Science, Amsterdam (1998). p.71-96. ISBN: 9780080527161.

[19] Fan Y, Nishida H, Shirai Y, Endo T. "Racemization on thermal degradation of poly(L-lactide) with calcium salt end structure". Polymer Degradation and Stability. 2003. Vol.80, p.503-511. http://dx.doi.org/10.1016/S0141-3910(03)00033-8

[20] Khabbaz F, Karlsson S, Albertsson AC. "Py-GC/MS an Effective Technique to Characterizing of Degradation Mechanism of Poly (L-lactide) in the Different Environment". Journal of Applied Polymer Science. 2000. Vol.78, p.2369-2378.

[21] Westphal C, Perrot C, Karlsson S. "Py-GC/MS as a means to predict degree of degradation by giving microstructural changes modelled on LDPE and PLA". Polymer Degradation and Stability. 2001. Vol.73 p.281-287. http://dx.doi.org/10.1016/S0141-3910(01)00089-1 